\title{
Variable-angle total internal reflection fluorescence microscopy of intact cells of Arabidopsis thaliana
}

\author{
Yinglang Wan', William M Ash $\mathrm{II}^{2}$, Lusheng Fan ${ }^{1,3}$, Huaiqin Hao ${ }^{1}$, Myung K Kim² and Jinxing Lin ${ }^{1 *}$
}

\begin{abstract}
Background: Total internal reflection fluorescence microscopy (TIRFM) is a powerful tool for observing fluorescently labeled molecules on the plasma membrane surface of animal cells. However, the utility of TIRFM in plant cell studies has been limited by the fact that plants have cell walls, thick peripheral layers surrounding the plasma membrane. Recently, a new technique known as variable-angle epifluorescence microscopy (VAEM) was developed to circumvent this problem. However, the lack of a detailed analysis of the optical principles underlying VAEM has limited its applications in plant-cell biology.

Results: Here, we present theoretical and experimental evidence supporting the use of variable-angle TIRFM in observations of intact plant cells. We show that when total internal reflection occurs at the cell wall/cytosol interface with an appropriate angle of incidence, an evanescent wave field of constant depth is produced inside the cytosol. Results of experimental TIRFM observations of the dynamic behaviors of phototropin 1 (a membrane receptor protein) and clathrin light chain (a vesicle coat protein) support our theoretical analysis.
\end{abstract}

Conclusions: These findings demonstrate that variable-angle TIRFM is appropriate for quantitative live imaging of cells in intact tissues of Arabidopsis thaliana.

Keywords: Quantitative, VA-TIRFM, optical analysis, intact cell, cell wall

\section{Background}

Total internal reflection fluorescence microscopy (TIRFM), also known as evanescent wave microscopy (EWM), is a powerful tool for observing the distribution and movement of fluorescently labeled molecules in an aqueous environment. This technique can be used when the molecules of interest are very close to the boundary between the aqueous environment and another medium with a higher refractive index $(n)$. It is based on the physical phenomenon of total internal reflection (TIR), which occurs when a ray of light strikes a boundary between two materials with different $n$ values and the incident angle $\left(\theta_{i}\right)$ is greater than the critical angle of incidence $\left(\theta_{c}\right)$ [1]. Under these conditions, all of the light is reflected back into the medium with the higher

\footnotetext{
* Correspondence: linjx@ibcas.ac.cn

${ }^{1}$ Key Laboratory of Plant Molecular Physiology, Institute of Botany, Chinese Academy of Sciences, Beijing 100093, China

Full list of author information is available at the end of the article
}

$n$ value (Formula 1) [1]. When TIR occurs, a nearfield light wave forms at the boundary; this "evanescent wave" (EW) can penetrate the surface of the medium to a depth $(d)$ approximately equal to $1 / 3$ of the wavelength of the incident light (Formula 2) [1].

Formula for calculating critical angle of incidence:

$$
\theta c=\sin ^{-1}\left(n_{1} / n_{2}\right)
$$

Formula for calculating the depth of an EW field:

$$
d=\lambda_{0} / 4 \pi\left(n_{2^{2}} \sin ^{2} \theta-n_{1^{2}}\right)^{-1 / 2}
$$

The TIRFM can provide a few hundred nanometers of excitation volume near the plasma membrane; this feature is particularly useful because the excitation volume is the site of many important events associated with the plasma membrane. TIRFM provides a higher spatial resolution and a higher signal-to-noise ratio than classic fluorescence microscopy or laser scanning confocal
C Biomed Central

(c) 2011 Wan et al; licensee BioMed Central Ltd. This is an Open Access article distributed under the terms of the Creative Commons Attribution License (http://creativecommons.org/licenses/by/2.0), which permits unrestricted use, distribution, and reproduction in any medium, provided the original work is properly cited. 
microscopy because the cytosol outside of the EW field remains unilluminated. When equipped with an electronmultiplier charge-coupled device (EM-CCD), the instrument can provide reliable imaging even with weak signals and a short exposure time. Therefore, the EM-CCD/ TIRFM combination is a viable solution for laboratories; it provides high spatial and temporal resolution for studying events occurring near the plasma membrane [2-4].

Because the TIRFM technique is unrivaled in its imaging capability in the near-boundary field, it has been successfully applied to in vivo studies of animal cells for the past decade $[3,4]$. However, the thick cell wall that surrounds the cytoplasm of plant cells has cast doubt on the possible utility of TIRFM in plant cell studies $[5,6]$. Recently, a new technique known as variable-angle epifluorescence microscopy (VAEM) was developed to circumvent this problem. The technique allows laser light to penetrate the cell wall using a sub-critical angle in which $\theta_{i}$ is slightly smaller than $\theta_{c}[7]$, and it has been used successfully in studies of the membrane physiology of plant cells $[8,9]$. However, because VAEM provides a relatively deep illumination field of variable depth, it is sometimes referred to as pseudo-TIRFM [5].

In the present study, we coupled our TIRFM equipment to a device that was used to vary $\theta_{i}$. Using this technique of variable-angle TIRFM (VA-TIRFM), we were able to observe the dynamic and endocytotic behaviors of green fluorescent protein (GFP)-tagged plant membrane proteins in living Arabidopsis thaliana cells. The specific membrane proteins examined were phototropin 1 (PHOT1; a photoreceptor protein) and clathrin light chain (CLC; an endosomal vesicle coat protein). During our experiments, we observed that PHOT1-GFP and CLC-GFP both exhibited two different modes of dynamic behavior, a lateral-movement mode and a blinking mode respectively. Observation of the blinking mode occurred only with true TIRFM, which provides an EW field with a constant depth of illumination. Therefore, we carried out an optical analysis to explain this experimental phenomenon and investigated whether VA-TIRFM will prove useful in the quantitative analysis of membraneassociated proteins in intact plant cells.

\section{Results and discussion}

Optical analysis of plant peripheral layers and limitations of VAEM in plant-cell studies

To analyze the optical principles underlying our fluorescence observations of plant cells, we first examined the peripheral layers around the plant cytosol. Unlike microscopic observations of animal cells (Figure 1A), microscopic observations of plant cells (Figure 1B) must accommodate five different peripheral layers between the incident light and the cytosol $(n=1.38)$; these layers are the immersion oil $(n=1.52)$, glass cover slip $(n=$ $1.52)$, aqueous medium $(n=1.33)$, cell wall $(n=1.42$ to $1.48)$, and plasma membrane $(d=7 \mathrm{~nm})[1,10]$. This complex optical layering causes two major difficulties in the application of TIRFM technology to plant cell studies. First, because the aqueous layer between the cell wall and the cover slip has the smallest refraction index of these peripheral layers, the interface between the glass and the aqueous medium (g/am interface) has the smallest $\theta_{c}$ of all the interfaces (Figure 2A). Second, because the cell wall is quite thick $(>200 \mathrm{~nm})$ [11], the depth of the EW field ( 100 nm) is insufficient to penetrate this thick boundary when TIR occurs at the g/am interface (Figure 2A). VAEM solves these problems by creating a sub-critical angle of incidence, resulting in a narrow illumination field inside cytosol Figure 1D and 2B) [7].

According to Formula 1, $\theta_{c}$ at the g/am interface is $61.0^{\circ}$. The angle of refraction $(\delta)$ inside the cytosol for a sub-critical angle of incidence $\left(\theta_{i}<\theta_{c}\right)$ can be calculated using Snell's law (Formulas 3 and 4). With the $n$ values given above for cytosol and glass and $\theta_{i}$ set at $60.0^{\circ}$ (slightly less than $\theta_{c}$ ), then $\delta$ is $72.5^{\circ}$ inside the cytosol. The depth of the illumination field from the refractive laser light can then be calculated by a trigonometric formula (Formula 5).

Snell's law describes the relationship between $\theta_{i}$ and $\delta$ as:

$$
\sin \theta_{i} / \sin \delta=n_{2} / n_{1}
$$

When incident light penetrates $\times$ different optical layers, $\delta$ in the last medium $\left(\delta_{x}\right)$ can be calculated as:

$$
\sin \theta_{i} / \sin \delta_{x}=n_{x} / n_{1}
$$

Variable depth of illumination (d) within an observation field (width = a) can be calculated as:

$$
d=a / \tan \delta_{x}
$$

In our experimental set-up, the observation field of the EM-CCD camera is $512 \times 512$ pixels, equivalent to $81.9 \times 81.9 \mu \mathrm{m}(a=81.9 \mu \mathrm{m})$; thus, according to Formula $5, d$ can vary from 0 to $25.9 \mu \mathrm{m}$ in this field (Figure $2 \mathrm{~B}$ and $2 \mathrm{E}$ ). Given that the width of a single cell is $20 \mu \mathrm{m}, d$ can vary from 0 to $6.3 \mu \mathrm{m}$. This theoretical result is consistent with the experimental results reported by Konopka et al., who used VAEM to observe GFP-tagged CLC and the GFP-tagged microtubule-binding domain in intact plant cells; they determined that the depth of the VAEM observation ranged from a few microns to approximately 40 microns [7]. Therefore, we have designated this illumination field as the variableangle epifluorescence (VAE) field (Figure 1D), distinct from the EW field associated with TIRFM (Figure 1C). 


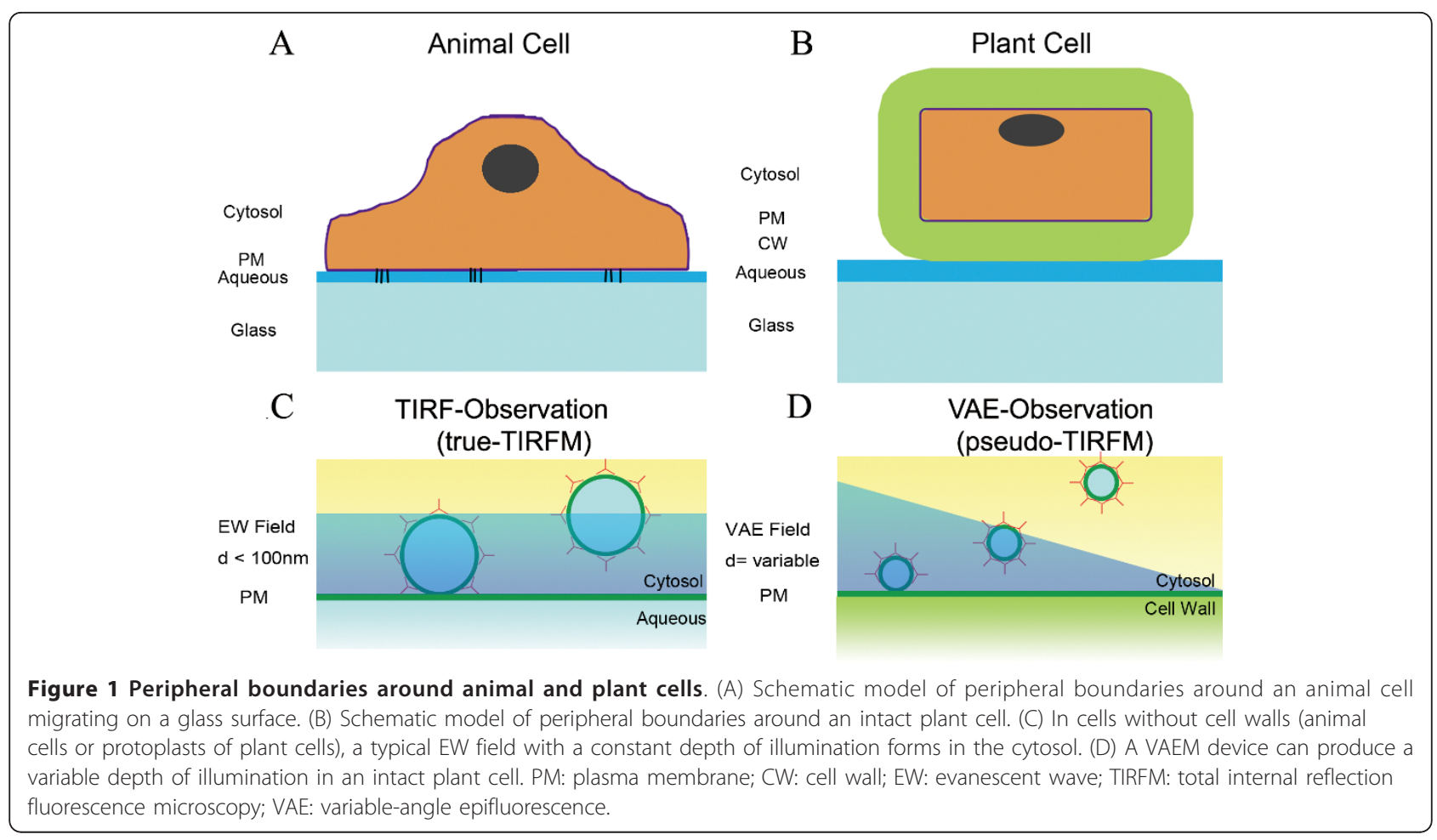

The narrow and constant EW illumination field is the major advantage of the TIRFM technique over laser scanning or spinning-disc confocal microscopy [3]. On the other hand, VAEM does not exceed these techniques in spatial resolution and is markedly limited in its usefulness for observations of intact plant cells because the variable illumination depth prevents accurate quantitation. The observed molecules do not maintain a constant dynamic state between different observations or among the different regions in a single observation with a variable illumination depth. In contrast, true TIRFM can be used to quantitatively measure the protein docking time on the plasma membrane [12] and to analyze the oligomeric states of membrane proteins in animal cells [13]. Thus, VAEM is inferior to true TIRFM; the disadvantages outlined above limit its suitability for studies of protein dynamics and behaviors in plant cell membranes.

\section{"Frustrated" TIR and EW field in intact plant cells}

Another major difference between plant and animal cells is that unlike migrating animal cells, plant cells do not adhere to glass surfaces. Thus, an aqueous film of varying thickness is present between the glass surface and the cell wall. When laser light strikes the g/am interface with $\theta_{i}>\theta_{c}$, and the aqueous film is sufficiently thin $(h<1 / 5 \lambda$; approximately $100 \mathrm{~nm}$ ), the tail of the evanescent wave transmits most of the light into the next medium so that only a small part of the light energy is reflected back; this phenomenon has been dubbed "frustrated" TIR (fTIR)
[14]. After calculating the altered $\theta_{c}$ and the loss of energy in the fTIR situation, Ash et al. used TIR holographic microscopy to examine onion epidermal cells attached to a glass surface [15]. Their analysis suggested that laser light can penetrate the aqueous medium even when $\theta_{i}$ $>\theta_{c}$. The critical factor for the fTIR phenomenon is a sufficiently thin aqueous film.

As shown in Figure 2C and 2D, a laser light beam can penetrate the aqueous medium under fTIR conditions. Thus, the first major obstacle to the application of TIRFM to plant-cell studies is overcome. If we consider the $n$ values of the different peripheral layers in plant cell microscopy, we see that TIR may occur at two different interfaces, the cell wall/cytoplasm (cw/c) interface and the glass/cell wall $(\mathrm{g} / \mathrm{cw})$ interface. Given that the average $n$ of cell walls is 1.45 [10] and that cell walls of outer epidermal cells are smooth and of constant thickness (roughly $250 \mathrm{~nm}$ ) [11], $\theta_{c}$ at the cw/c interface $\left(\theta_{c(c w / c)}\right)$ is $72.1^{\circ}$. According to Snell's law (Formula 3), $\theta_{i}$ from the objective lens $\left(\theta_{c 1}\right.$ in Figure $2 \mathrm{C}$ and $\left.2 \mathrm{E}\right)$ should be greater than $65.2^{\circ}$ to create TIR at the cw/c interface. When $\theta_{i}$ is further increased by the variable angle device and reaches $\theta_{c}$ at the $\mathrm{g} / \mathrm{cw}$ interface $\left(\theta_{c(g / c w)}=72.5^{\circ}\right)$, the light beam is completely reflected back $\left(\theta_{c 2}\right.$ in Figure $\left.2 \mathrm{D}\right)$. Again, the EW field cannot reach the cytosol in an intact cell. There are two critical requirements to obtain EW field illumination inside the cytosol in an intact plant cell: an incident angle between $\theta_{c 1}$ and $\theta_{c 2}\left(65.2^{\circ}<\theta_{i}<72.5^{\circ}\right)$ and a sufficiently thin aqueous film $(d<100 \mathrm{~nm})$. When the EW 


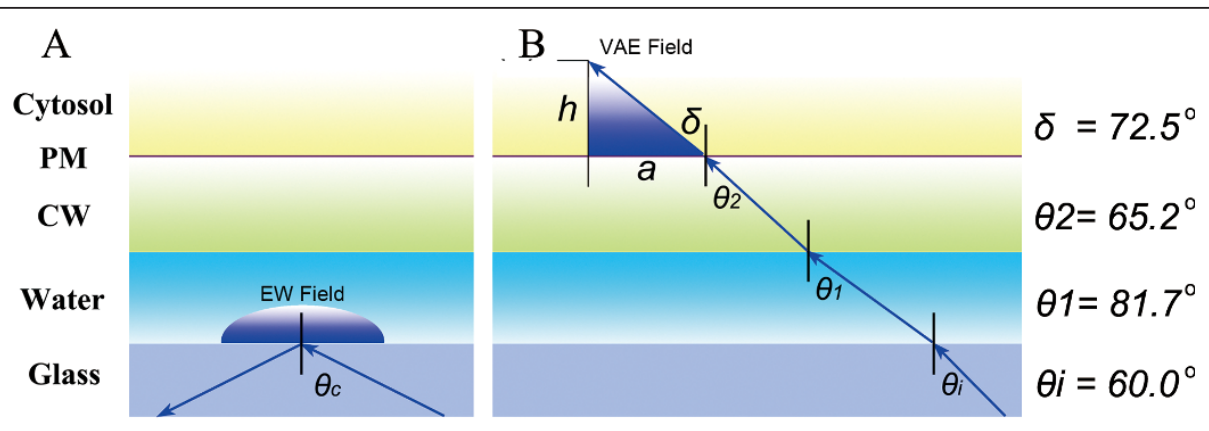

$\mathrm{C}$

Cytosol

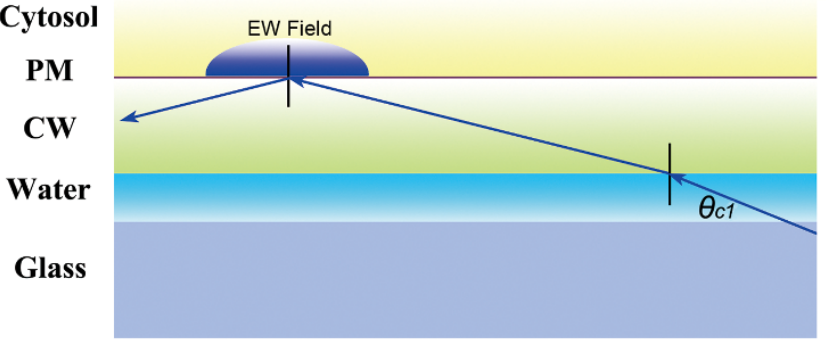

$\mathrm{D}$

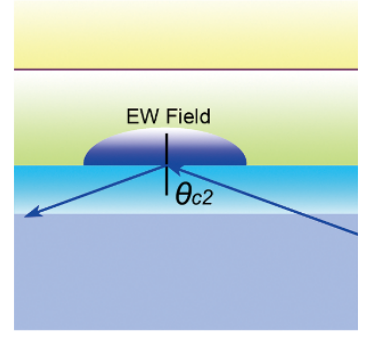

$\mathrm{E}$

\begin{tabular}{|c|cccc|}
\hline & $\begin{array}{c}\text { Refractive } \\
\text { index }\end{array}$ & $\begin{array}{c}\text { Thickness } \\
(\mathrm{nm})\end{array}$ & $\begin{array}{c}\text { Critical } \\
\text { angle }\end{array}$ & $\begin{array}{c}\text { Depth } \\
\text { of EW }\end{array}$ \\
\hline Glass & 1.52 & $140 \mu \mathrm{m}$ & & - \\
Water & 1.33 & $>100 \mathrm{~nm}$ & $\theta_{c}: 61.0^{\circ}$ & $105 \mathrm{~nm}$ \\
\hline Water & & $<100 \mathrm{~nm}$ & - & - \\
Cell Wall & $1.42-1.48$ & $250 \mathrm{~nm}$ & $\theta_{c 1}: 72.5^{\circ}$ & - \\
Cytosol & 1.38 & - & $\theta_{c 2}: 65.2^{\circ}$ & $159 \mathrm{~nm}$ \\
\hline
\end{tabular}

Figure 2 Optical analysis of light paths in VAEM and TIRFM observations in plant cells. (A) When the aqueous film between the cover glass and the cell wall is thick $(>100 \mathrm{~nm})$ and $\theta_{i}$ is greater than $61.0^{\circ}\left(\theta_{c}\right)$, all light energy is reflected at the g/am interface. The EW field forms in the aqueous medium but cannot penetrate into the cytosol. (B) When $\theta_{i}$ is less than $61.0^{\circ}\left(\theta_{c}\right)$, a VAE field forms, illuminating the cytosol with variable depth. Angles of refraction for all optical boundaries with $\theta_{i}=60.0^{\circ}$ are shown. (C) When the aqueous film is sufficiently thin ( $<100 \mathrm{~nm}$ ) and $\theta_{i}$ is smaller than $\theta_{c}$ for $\mathrm{g} / \mathrm{cw}\left(\theta_{c 2}\right)$ but greater than $\theta_{c}$ for $\mathrm{g} / \mathrm{c}\left(\theta_{c 1}\right)$ [(i.e., $\left.\theta_{c 1}<\theta_{i}<\theta_{c 2}\right)$ ], an EW field of constant depth is created inside the cytosol at the cw/c interface. (D) When $\theta_{i}>\theta_{c 2}$, the light is completely reflected at the cell wall interface, and the EW field is not deep enough to penetrate into the cytosol. (E) Optical parameters of plant-cell peripheral boundaries. In the situation shown in (A), the EW field is $105 \mathrm{~nm}$ deep and cannot penetrate the cell wall. In the situation shown in (C) and with $\theta_{i}=67.0^{\circ}$, the EW field penetrates $159 \mathrm{~nm}$ into the cytosol. CW: cell wall; EW: evanescent wave; PM: plasma membrane; VAE: variable-angle epifluorescence.

field occurs inside the cytosol of an intact plant cell, the depth of illumination is constant for a single observation (Figure 2E).

Cell walls can be removed through enzymatic digestion to create protoplasts, "naked" plant cells that provide another approach for obtaining EW field illumination inside the plant cell cytosol. When protoplasts are tightly attached to a glass surface, the EW field can penetrate into the cytosol in a manner similar to that in TIRFM observation of animal cells (Figure 1C). However, the preparation of protoplasts seems likely to affect the organization of at least some membrane and cytoskeletal proteins $[16,17]$, limiting the usefulness of protoplasts in physiological studies.

EW and VAE field observations of PHOT1-GFP and CLCGFP in intact plant cells

To test our theoretical analysis of EW and VAE field observation, we performed TIRFM and VAEM experiments. The results were unambiguous and reproducible. For example, when we examined GFP-tagged PHOT1 or CLC in transgenic Arabidopsis cells using VA-TIRFM, 
we observed two different dynamic modes: a lateralmovement mode (Figure $3 \mathrm{~A}$ and $3 \mathrm{~B}$ ), additional movie files show this mode in more detail (see Additional file 1 and 2 online); and a blinking mode (Figure 3C and 3D), additional movie files show this mode in more detail (see Additional file 3 and 4 online). Because a uniformly present blinking mode can only be observed in an EW field with a constant depth of illumination, our observation of a blinking mode was a true-TIRFM observation. (Actually, it was this original observation that prompted us to analyze the optical characteristics of the peripheral layers in plant-cell microscopy. In turn, these theoretical analyses of the fTIR phenomenon have supported our TIRFM observations in intact plant tissues).

PHOT1 is a membrane-associated receptor of blue light that localizes on the peripheral membrane of hypocotyl cortical and epidermal cells [18]. In 4-day-old dark-grown seedlings, PHOT1-GFP is found on the plasma membrane, but exposure to blue light initiates its internalization by activating its functional domains $[18,19]$. In our experiments, we found that the lateral-movement mode of PHOT1-GFP was observable only after a 3-5-min period

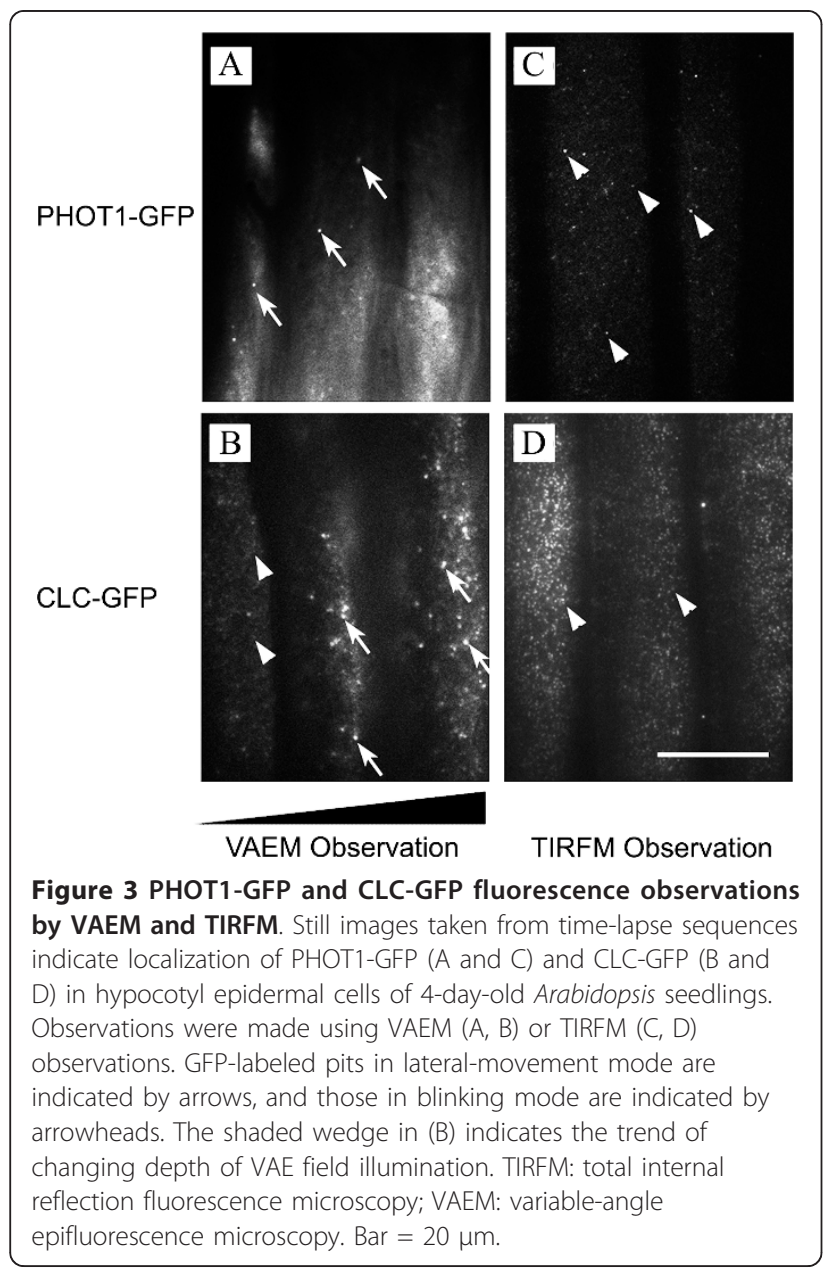

of blue laser illumination (Figure 3A and Additional file 1 online), suggesting that the PHOT1-GFP spots moving laterally were blue-light-induced PHOT1-GFP endosomes moving inside the cell near the plasma membrane (i.e., in the VAE field).

When we changed the $\theta_{i}$ so that $\theta_{c 1}<\theta_{i}<\theta_{c 2}$ (Figure 2C) and gently pressed the seedling against the surface of the glass slide, we observed a typical TIRFM image showing blinking behavior of the labeled protein (Figure $3 \mathrm{C}$ and Additional file 3 online). This finding confirms the theoretical analysis above, showing that with a sufficiently thin water film and a carefully adjusted $\theta_{i}$, membrane receptor proteins can also be observed in the EW field (Figure 2C). In comparing the dynamic modes of PHOT1-GFP molecules, we found that the PHOT1-GFP spots exhibiting lateral movement were both more numerous and more intense than those exhibiting blinking behavior. This observation suggested that some of the PHOT1-GFP was assembled in early endosomes or the trans-Golgi network, whereas the PHOT1-GFP in the plasma membrane was distributed as monomers or dimers (Kaiserli et al. 2009). Together, these observations of PHOT1-GFP provide solid evidence supporting our current functional model of VA-TIRFM observation in intact plant cells (Figure 2C).

This functional model was also confirmed by our observations of the dynamic modes of CLC-GFP in an intact Arabidopsis seedling. CLC molecules normally assemble together with clathrin heavy chains to form a "cage" that coats endosomal vesicles [20]. The docking time of CLC-GFP signals at the plasma membrane may reflect the maturation phase of clathrin-coated pits $[12,21]$. Because many CLC-GFP molecules interact during cage assembly on the plasma membrane, whereas PHOT1 exists only as monomers and dimers on the plasma membrane $[19,20]$, the CLC-GFP and PHOT1GFP spots were expected to be of different sizes and to reflect different dynamic behaviors (Figure 3). Nonetheless, as we observed for PHOT1-GFP, CLC-GFP also exhibited both blinking and lateral-movement modes. Interestingly, the blinking mode (arrowheads in Figure 3B) and the lateral-movement mode (arrows in Figure $3 \mathrm{~B}$ ) were both observed in the same visual field (Additional file 2 online), indicating a highly variable depth for the VAE field. In the regions with more shallow illumination, most of the CLC-GFP spots were blinking, with only a few laterally moving spots present in the same cell (Figure 3B, left). In the regions with deep illumination fields, more laterally moving CLC-GFP spots were found (Figure 3B, right). In contrast, when cells were observed in a constant EW field, no laterally moving spots were detected in the entire visual field (Figure $3 \mathrm{D}$ and Additional file 4 online). Taken together, these experimental results constitute strong evidence that 
true-TIRFM observations can be made in intact plant cells. Therefore, these observations are a valid basis for quantitative analyses.

\section{Conclusions}

Our theoretical analyses and experimental results demonstrate that the $\theta_{i}$ selected on VA-TIRFM equipment and the firmness of attachment of intact seedlings to the glass cover slip affect the type of observation of GFP-tagged membrane proteins that is made. Observation of lateral movement indicates VAEM, whereas observation of blinking behavior indicates a true TIRFM (EWM). These two clearly distinguishable results support the validity of quantitative analysis of VA-TIRFM results. Trajectory-tracking methods [22-24] can be used with VA-TIRFM to record the parameters of molecule movement, such as velocity and mean square displacement, allowing their roles in cellular trafficking and signaling processes to be analyzed. Furthermore, fluctuation in the observed fluorescence intensity might reflect movement through early endosomes or the trans-Golgi network from the plasma membrane [25]. Analyses of attachment time and photobleaching of blinking spots can be used to quantitatively determine the attachment mode, endocytosis rate, and oligomeric states of membrane-attached proteins $[13,25,26]$.

\section{Methods}

Plant material

The Arabidopsis seedlings expressing $P R E_{P H O T 1}:: P H O T 1-$ GFP in the phot1-5 background [27] and PRE $C L C:: C L C$ GFP in WT (Col-0) background [8] were observed in this study. The seeds were sterilized and planted on the surface of $0.4 \%$ phytagel $^{\circledR}$ (Sigma-Aldrich, St. Louis, MO. USA) medium with 1/2 MS basal salts (Sigma-Aldrich) and 1\% sucrose (Sigma-Aldrich). The seedlings were put in the chamber with illumination from white fluorescence lamps with 7000-10000 lux of illuminance for 16 hours per day at a temperature of $22^{\circ} \mathrm{C}$.

\section{VA-TIRFM setup and observation}

An objective-type TIR fluorescence microscope was built using an inverted microscope (IX-71, Olympus) equipped with a $100 \times$ oil-immersion objective lens (numerical aperture $=1.45$ ) and a diode laser (Changchun New Industries Optoelectronics Tech. Co., Ltd., Changchun, China) for illumination of objects through an adjustable-angle internal reflective fluorescence illuminator. GFP-tagged molecules were excited by a $471 \mathrm{~nm}$ laser, respectively, and fluorescence signals were collected by the objective. Signals were filtered through either an FF01-525/45-25 band-pass filter (Semrock, Lake Forest, Illinois, USA) and then directed using a back-illuminated EM-CCD camera (ANDOR iXon DV8897D-CS0-VP, Andor Technology plc., Belfast, Northern Ireland). The Micro-Manager program (version 1.3; open source software developed by the Vale Lab at the University of California, San Francisco, CA, USA) was used to operate the equipment. For sample preparation, Arabidopsis seedlings were immersed in halfstrength Murashige and Skoog liquid medium on a cover glass (BRAND Gmbh, Wertheim, Germany; $n, 1.52 \pm 0.01$; thickness, $0.13-0.17 \mathrm{~mm}$ ). Another cover glass was placed on top of the sample, and this sandwich was pressed gently to tightly attach the seedling to the glass surface.

\section{Additional material}

Additional file 1: All these additional video files are reconstructed
from time lapse sequences with 300 images in a 60 fps frame rate.
The exposure time for each image is 100 ms, interval time between
images is $50 \mathrm{~ms} .1$ pixel $=0.16 \mu \mathrm{m}$. VAEM observation of PHOT1-GFP
(Pseudo-TIRFM).
Additional file 2: All these additional video files are reconstructed
from time lapse sequences with 300 images in a 60 fps frame rate.
The exposure time for each image is 100 ms, interval time between
images is 50 ms. 1 pixel $=0.16 \mu \mathrm{m}$. VAEM observation of CLC-GFP
(Pseudo-TIRFM).
Additional file 3: All these additional video files are reconstructed
from time lapse sequences with 300 images in a 60 fps frame rate.
The exposure time for each image is 100 ms, interval time between
images is 50 ms. 1 pixel = $0.16 \mu \mathrm{m}$. TIRFM observation of PHOT1-GFP
(True-TIRFM).
Additional file 4: All these additional video files are reconstructed
from time lapse sequences with 300 images in a 60 fps frame rate.
The exposure time for each image is 100 ms, interval time between
images is 50 ms. 1 pixel = $0.16 \mu m$. TIRFM field observation of CLC-GFP
(True-TIRFM).

List of abbreviations

CLC: clathrin light chain; CW: cell wall; cw/c: cell wall/cytoplasm interface; EM-CCD: electron-multiplier charge-coupled device; EW: evanescent wave; EWM: evanescent wave microscopy; fTIR: "frustrated" TIR; g/am: glass/ aqueous medium interface; $\mathbf{g} / \mathbf{c w}$ : glass/cell wall interface; GFP: green fluorescent protein; PHOT: phototropin; PM: plasma membrane; TIR: total internal reflection; TIRFM: total internal reflection fluorescence microscopy; VAE: variable-angle epifluorescence; VAEM: variable-angle epifluorescence microscopy; VA-TIRFM: variable-angle total internal reflection fluorescence microscopy.

\section{Acknowledgements}

We thank Prof. Dr. Winslow R Briggs (Carnegie Institution for Science, Department of Plant Biology) for providing us with seeds of PHOT1-GFP transformed Arabidopsis line. We also thank Prof. Dr. Sebastian Y. Bednarek (Department of Biochemistry, University of Wisconsin-Madison) for providing us with seeds of CLC-GFP transformed Arabidopsis line. This work is supported by the National Basic Research Program of China (973 Program 2011 CB809103 and 2007CB108703), the CAS/SAFEA International Partnership Program for Creative Research Teams (20090491019), the National Natural Science Foundation of China (31000595, 30730009, 30821007 and 30900072), the Knowledge Innovation Program of the Chinese Academy of Sciences (KJCX2-YW-L08, KSCX2-EW-J-1) and from China Postdoctoral Science Foundation.

\section{Author details}

${ }^{1}$ Key Laboratory of Plant Molecular Physiology, Institute of Botany, Chinese Academy of Sciences, Beijing 100093, China. ${ }^{2}$ Digital Holography and Microscopy Laboratory, Department of Physics, University of South Florida, Tampa, Florida 33620, USA. ${ }^{3}$ Gradual School of Chinese Academy of Sciences, Beijing, 100049, China. 


\section{Authors' contributions}

YW performed the optical analysis, wrote the manuscript. LF and $\mathrm{HH}$ provided the experimental results. WMAll and MKK proved the optical principles and provided important and essential suggestions on writing of this manuscript. JL supervised this project. All authors read and approved the final manuscript.

\section{Competing interests}

The authors declare that they have no competing interests.

Received: 24 July 2011 Accepted: 24 September 2011

Published: 24 September 2011

\section{References}

1. Fish K: Total internal reflection fluorescence (TIRF) microscopy. Curr Protoc Cytom 2009, 12:Unit 12.18.

2. Groves JT, Parthasarathy R, Forstner MB: Fluorescence imaging of membrane dynamics. Annu Rev Biomed Eng 2008, 10:311-338.

3. Mashanov Gl, Tacon D, Knight AE, Peckham M, Molloyc JE: Visualizing single molecules inside living cells using total internal reflection fluorescence microscopy. Methods 2003, 29:142-152.

4. Sako Y, Minoguchi S, Yanagida T: Single-molecule imaging of EGFR signaling on the surface of living cells. Nat Cell Biol 2000, 2:168-172.

5. Gutierrez R, Grossmann G, Frommer WB, Ehrhardt DW: Opportunities to explore plant membrane organization with super-resolution microscopy. Plant Physiol 2010, 154:463-466.

6. Tanner W, Malinsky J, Opekarová M: In plant and animal cells, detergentresistant membranes do not define functional membrane rafts. Plant Cell 2011, 23:1191-1193.

7. Konopka CA, Bednarek SY: Variable-angle epifluorescence microscopy: a new way to look at protein dynamics in the plant cell cortex. Plant $J$ 2008, 53:186-196.

8. Konopka CA, Backues SK, Bednarek SY: Dynamics of Arabidopsis dynaminrelated protein $1 \mathrm{C}$ and a clathrin light chain at the plasma membrane. Plant Cell 2008, 20:1363-1380

9. Konopka CA, Bednarek SY: Comparison of the dynamics and functional redundancy of the arabidopsis dynamin-related isoforms DRP1A and DRP1C during plant development. Plant Physiol 2008, 147:1590-1602.

10. Woolley JT: Refractive index of soybean leaf cell walls. Plant Physiol 1975, 55:172-174.

11. Derbyshire P, Findlay K, McCann MC, Robert K: Cell elongation in Arabidopsis hypocotyls involves dynamic changes in cell wall thickness. J Exp Bot 2007, 58:2079-2089.

12. Loerke D, Mettlen M, Yarar D, Jaqaman K, Jaqaman H, Danuser G, Schmid SL: Cargo and dynamin regulate clathrin-coated pit maturation. PLoS Biol 2009, 7:e1000057.

13. Zhang W, Jiang Y, Wang Q, Ma X, Xiao Z, Zuo W, Fang X, Chen Y: Singlemolecule imaging reveals transforming growth factor-b-induced type II receptor dimerization. Proc Natl Acad Sci USA 2009, 106:15679-15683.

14. Rivière D: An optical study of thin interfacial liquid films using total reflection of light. Exp Fluids 1987, 5:349-354.

15. Ash WM III, Krzewina L, Kim MK: Quantitative imaging of cellular adhesion by total internal reflection holographic microscopy. Appl Opt 2009, 48:144-152.

16. Cleary AL: Plasma membrane-cell wall connections: Roles in mitosis and cytokinesis revealed by plasmolysis of Tradescantia virginiana leaf epidermal cells. Protoplasma 2001, 215:21-34.

17. Wojtaszek P, Baluska F, Kasprowicz A, Luczak M, Volkmann D: Domainspecific mechanosensory transmission of osmotic and enzymatic cell wall disturbances to the actin cytoskeleton. Protoplasma 2007 230:217-230

18. Wan Y, Eisinger W, Ehrhardt D, Kubitscheck U, Baluska F, Briggs W: The subcellular localization and blue-light-induced movement of phototropin 1-GFP in etiolated seedlings of Arabidopsis thaliana. Mol Plant 2008, 1:103-117.

19. Kaiserli E, Sullivan S, Jones MA, Feeney KA, Christie JM: Domain swapping to assess the mechanistic basis of Arabidopsis phototropin 1 receptor kinase activation and endocytosis by blue light. Plant Cell 2009, 21:3226-3244.

20. Kirchhausen T: Imaging endocytic clathrin structures in living cells. Trends Cell Biol 2009, 19:596-605.
21. Ryan TA: Kiss-and-run, fuse-pinch-and-linger, fuse-and-collapse: The life and times of a neurosecretory granule. Proc Natl Acad Sci USA 2003, 100:2171-2173.

22. Jagaman K, Loerke D, Mettlen M, Kuwata H, Grinstein S, Schmid SL, Danuser G: Robust single-particle tracking in live-cell time-lapse sequences. Nat Methods 2008, 5:695-702.

23. Wang X, Teng Y, Wang Q, Li X, Sheng X, Zheng M, Šamaj J, Baluška F, Lin J: Imaging of dynamic secretory vesicles in living pollen tubes of Picea meyeri using evanescent wave microscopy. Plant Physiol 2006, 141:1591-1603.

24. Zheng $M$, Beck M, Müller J, Chen T, Wang $X$, Wang F, Wang $Q$, Wang $Y$, BalušKa F, Logan DC, Šamaj J, Lin J: Actin turnover is required for myosindependent mitochondrial movements in Arabidopsis root hairs. PLOS ONE 2009, 4:e5961.

25. Veliz LA, Toro CA, Vivar JP, Arias LA, Villegas J, Castro MA, Brauchi S: NearMembrane Dynamics and Capture of TRPM8 Channels within Transient Confinement Domains. PLOS ONE 2010, 5:e13290.

26. Hern JA, Baig AH, Mashanov Gl, Birdsall B, Corrie JE, Lazareno S, Molloy JE, Birdsall NJ: Formation and dissociation of M1 muscarinic receptor dimers seen by total internal reflection fluorescence imaging of single molecules. Proc Natl Acad Sci USA 2010, 107:2693-2698.

27. Sakamoto K, Briggs WR: Cellular and subcellular localization of phototropin 1. Plant Cell 2002, 14:1723-1735.

\section{doi:10.1186/1746-4811-7-27}

Cite this article as: Wan et al:: Variable-angle total internal reflection fluorescence microscopy of intact cells of Arabidopsis thaliana. Plant Methods 2011 7:27.

\section{Submit your next manuscript to BioMed Central and take full advantage of:}

- Convenient online submission

- Thorough peer review

- No space constraints or color figure charges

- Immediate publication on acceptance

- Inclusion in PubMed, CAS, Scopus and Google Scholar

- Research which is freely available for redistribution

Submit your manuscript at www.biomedcentral.com/submit
C Biomed Central 